Nonlinear Processes in Geophysics (2003) 10: 453-461

\title{
Response of a global atmospheric circulation model to spatio-temporal stochastic forcing: ensemble statistics
}

\author{
V. Pérez-Muñuzuri ${ }^{1}$, M. N. Lorenzo ${ }^{1}$, P. Montero ${ }^{1}$, K. Fraedrich $^{2}$, E. Kirk ${ }^{2}$, and F. Lunkeit ${ }^{2}$ \\ ${ }^{1}$ Group of Nonlinear Physics, Faculty of Physics, University of Santiago de Compostela. E-15782 Santiago de Compostela, \\ Spain \\ ${ }^{2}$ Meteorological Institute, University of Hamburg, Bundesstr. 55, D-20146 Hamburg, Germany
}

Received: 23 September 2002 - Revised: 2 September 2003 - Accepted: 13 October 2003

\begin{abstract}
The response of a simplified global atmospheric circulation model (PUMA) to spatiotemporal stochastic forcing is analyzed using the statistical measures originally developed for ensemble forecast evaluation. The nontrivial effects of time and length correlations of the stochastic forcing on the ensemble scores (e.g. spread and 'error') are studied. A maximum for these scores is observed to occur for specific values of the correlation time. The effects of multiplicative and additive contributions of the correlated noise are analyzed in terms of the noise and PUMA parameters.
\end{abstract}

\section{Introduction}

Uncertainties in initial conditions and model parameterizations onto flow-dependent instabilities of the atmospheric processes affect predictability. The problem of predicting uncertainty can be solved in a probabilistic framework in terms of the Liouville equation for the initial uncertainties or a form of Fokker-Plank equation if model errors are also considered. However, such approaches are not computationally feasible in a realistic time. The solution passes through ensembles of integrations of meteorological models, with suitable perturbations to initial conditions and model parameterizations. In this way, the time evolution of the model needs to be considered in probabilistic terms.

Since a decade operational ensemble forecasts in Numerical Weather Prediction (NWP) have been introduced and the most suitable choice of initial perturbations has dominated the research. This is an important problem in the perfect model environment, but it may be less relevant for ensemble forecasts in the imperfect model scenario with systematic model errors (for simple models see Smith et al., 1999; Fraedrich and Ziehmann-Schlumbohm, 1994). While uncertainty in initial conditions matters in the first approach towards ensemble prediction, ensembles defined by multi-

Correspondence to: V. Pérez-Muñuzuri

(vicente.perez@cesga.es) model predictions account for the possible model errors. A first comparison between these two basically different ensemble prediction schemes reveals that, despite the larger ensemble size, the quality of the single NWP ensemble is not superior to that of the multi-model ensemble (Ziehmann, 2000). Multi-model ensembles incorporating a stochastic representation of model error have proven to be of greater value than ensembles based on perturbation at the initial conditions at the medium range (Buizza et al., 1999; Harrison et al., 1999), suggesting than model uncertainty can be used advantageously in an ensemble approach. In fact, the use of stochastic noise to represent unpredictable small-scale variability is familiar in a number of geophysical models (Hasselmann, 1976; Pitcher, 1977; Farrell and Ioannou, 1993; DelSole and Farrell, 1995; Penland, 1996; Newman et al, 1997; Moore and Kleeman, 1999; Palmer, 2001; Mylne et al, 2002; Barkmeijer et al., 2003).

The use of ensembles for seasonal to interannual forecasts has become a major advance in recent years (Anderson et al, 1999; Kalnay, 2003). Slowly varying surface forcing can produce atmospheric anomalies that are longer lasting and more predictable than individual patterns. The most notable of these is the El Niño Southern Oscillation (ENSO) produced by unstable oscillations of the coupled ocean-atmosphere system (Cane et al., 1986). Thus, for seasonal forecasts, a single atmospheric forecast forced with predicted sea surface temperatures (SST) anomalies would not be useful beyond the Lorenz's two week limit of weather predictability, while ensemble averaging many forecasts made with atmospheric models forced by SST anomalies allows the improvement of the forecast.

The necessity to consider model deficiencies and enhancing the size of the multi-model ensemble for seasonal and weather forecast initiated our study. The following analysis is confined to the response of an atmospheric circulation model on additive and multiplicative spatiotemporal stochastic forcing in terms of time averaging of ensemble means, representing imperfect multi-model ensembles.

The role of spatiotemporal stochastic forcing was previ- 
ously analyzed in simple nonlinear models of reduced dimensionality, where only local coupling among grid points was considered (Lorenzo and Pérez-Muñuzuri, 1999, 2001; Lorenzo et al., 2003). Following this idea, we have perturbed stochastically a simplified atmospheric global circulation model, PUMA (Portable University Model of the Atmosphere) developed at Hamburg University (Fraedrich et al., 1998). In this case, we want to confirm the role of correlated noise on a more complete model where nonlinear interactions, advection and diffusion terms are considered. Noise correlations are chosen so that non trivial effects can be obtained. Forcing increases the spread of the ensemble for a certain value of the correlation time where the predictability also attains a critical value. On the other hand, for increasing correlation length, the numerical results suggest a monotonous behavior of the ensemble spread. The influence of the effect of the multiplicative or additive contributions of the noise is also shown. The introduction of randomness in the physics of the model has a very good impact on the ensemble since it increases the ensemble spread to levels higher than those obtained when considering only an ensemble based on perturbations to the initial field.

Section 2 introduces the circulation model, its diabatic parameterization, a model climatology and the spatiotemporal stochastic forcing. The design of the simulations and their evaluation is described in Sect. 3, followed by the presentation of the results in Sect. 4. The conclusion (Sect. 5) notes the implications for ensemble predictions.

\section{The model}

The PUMA is a multi-level spectral model with triangular truncation using the semi-implicit time integration as described by (Hoskins and Simmons, 1975). Absolute vorticity $(\zeta)$, horizontal divergence $(D)$, temperature $(T)$, and the logarithm of the surface pressure $\left(\ln p_{s}\right)$ are the prognostic variables. Terrain following $\sigma$-coordinates $\left(\sigma=p / p_{s}\right)$ are used in the vertical. The dynamical core is forced by Newtonian cooling and Rayleigh friction. Then, PUMA equations can be written as,

$$
\frac{\partial \zeta}{\partial t}=\frac{1}{1-\mu^{2}} \frac{\partial}{\partial \lambda} F_{v}-\frac{\partial}{\partial \mu} F_{u}-\frac{\xi}{\tau_{f}}-K \nabla^{8} \xi
$$

$$
\begin{aligned}
\frac{\partial D}{\partial t} & =\frac{1}{1-\mu^{2}} \frac{\partial}{\partial \lambda} F_{u}+\frac{\partial}{\partial \mu} F_{v} \\
& -\nabla^{2}\left(\frac{U^{2}+V^{2}}{2\left(1-\mu^{2}\right)}-\Phi+\bar{T} \ln p_{s}\right) \\
& -\frac{D}{\tau_{f}}-K \nabla^{8} D,
\end{aligned}
$$

$$
\begin{aligned}
\frac{\partial T^{\prime}}{\partial t} & =-\frac{1}{1-\mu^{2}} \frac{\partial}{\partial \lambda}\left(U T^{\prime}\right)-\frac{\partial}{\partial \mu}\left(V T^{\prime}\right) \\
& +\left[1+\eta_{m}(\lambda, \phi ; t)\right] D T^{\prime}
\end{aligned}
$$

$$
\begin{aligned}
&- \dot{\sigma} \frac{\partial T}{\partial \sigma}+\kappa \frac{T w}{p}+\frac{T_{R}-T}{\tau_{c}}+ \\
& \eta_{a}(\lambda, \phi ; t)-K \nabla^{8} T^{\prime}, \\
& \frac{\partial\left(\ln p_{s}\right)}{\partial t}=-\frac{U}{1-\mu^{2}} \frac{\partial\left(\ln p_{s}\right)}{\partial \lambda}-V \frac{\partial\left(\ln p_{s}\right)}{\partial \mu}-D \\
&-\frac{\partial \dot{\sigma}}{\partial \sigma}, \\
& \frac{\partial \Phi}{\partial(\ln \sigma)}=-T,
\end{aligned}
$$

with

$F_{u}=V \zeta-\dot{\sigma} \frac{\partial U}{\partial \sigma}-T^{\prime} \frac{\partial\left(\ln p_{s}\right)}{\partial \lambda}$

$F_{v}=U \zeta-\dot{\sigma} \frac{\partial V}{\partial \sigma}-T^{\prime}\left(1-\mu^{2}\right) \frac{\partial\left(\ln p_{s}\right)}{\partial \mu}$

where $\xi$ denotes relative vorticity and temperature is expressed as $T=\bar{T}(\sigma)+T^{\prime}$ where $T^{\prime}$ is the fluctuating component. $\lambda$ and $\phi$ are longitude and latitude respectively, $\mu=\sin \phi, \Phi$ is the geopotential, $\kappa$ is the adiabatic coefficient, $w$ is the vertical velocity, $U=u \cos \phi=u \sqrt{1-\mu^{2}}$, $V=v \cos \phi=v \sqrt{1-\mu^{2}}(u, v$ are the zonal and meridional velocities) and $K$ a diffusion coefficient. The hyperdiffusion term $\left(\propto \nabla^{8}\right)$ represents the effect of subgrid scale eddies. Equations (1) and (2) are for the vorticity and divergence, respectively. Equation (3) is the thermodynamic equation, Eq. (4) is the conservation of mass (continuity) and Eq. (5) expresses the hydrostatic equilibrium in $\sigma$ coordinates. All simulations described below use the same parameter constellation (Lunkeit, 2001). For our simulations, we chose five vertical levels, a T21 horizontal resolution (i.e. approximately $5.6^{\circ} \times 5.6^{\circ}$ on the corresponding Gaussian grid), and a time step of half an hour.

Diabatic processes and annual cycle: Radiative and convective heating are described by a Newtonian cooling term $\left(T_{R}-T\right) / \tau_{c}$ in the temperature equation. This term forces the model temperature $T$ to relax toward the temperature profile $T_{R}$ with characteristic time scale $\tau_{c}$. The temperature profile $T_{R}(\lambda, \phi, \sigma ; t)$ represents the equilibrium profile induced by solar heating which is allowed to vary periodically in time with a period of $T_{a c}$ days as,

$T_{R}=\bar{T}_{R}+\hat{T_{R}} \cos \left(\frac{2 \pi}{T_{a c}} t+P_{a c}\right)$

where $\bar{T}_{R}$ is the mean of the restoration temperature field, while $\hat{T}_{R}$ and $P_{a c}$ represent the amplitude and phase of the cycle, respectively ${ }^{1}$.

Model variability and climate: In the adiabatic version, models of the PUMA type have been used to simulate and analyze growth and decay of large scale synoptic disturbances in the midlatitude atmosphere (Hoskins and Simmons, 1975).

\footnotetext{
${ }^{1}$ In this paper, the usual one year value of $T_{a c}$ is changed for research purposes.
} 
In the diabatic version with Newtonian cooling and Rayleigh friction, the model simulates the internal variability of the general circulation and its climate mean state. The general set up of the simulations described below follows Lunkeit (2001): The distribution of the relaxation temperature for the Newtonian cooling is zonally symmetric with an annual mean equator pole difference $\bar{T}_{R}$ of $70 \mathrm{~K}$. Idealized annual cycles with a phase $P_{a c}$ of zero days and different periods $T_{a c}$ are superimposed using an amplitude $\hat{T}_{R}$ of $60 \mathrm{~K}$. A lapse rate of $0.0065 \mathrm{~K} / \mathrm{m}$ defines the vertical temperature profile in the troposphere. The Newtonian cooling relaxation time scale, $\tau_{c}$, is 30 days for all levels. Rayleigh friction is confined to the lowermost level with a time scale $\tau_{f}$ of one day. The hyperdiffusion acts on all waves and damps the smallest resolved wave with a time scale of 0.25 days. For the chosen parameter setting the model climate is illustrated in Fig. 1. It shows a cross section of the zonal time mean zonal wind (a) and the power spectrum of the global mean angular velocity (b), which is positive thus indicating superrotation of the atmosphere. The overall slope of the spectrum is somewhat steeper than a red noise power law with enhanced variability on the timescales of synoptic activity (5-50 days). This spectrum is similar for a wide range of parameter values. In summarizing, PUMA is sufficiently representative to describe the internal dynamics and the mean state of the Earth's general circulation.

Spatiotemporal noise: The multiplicative and additive spatiotemporal stochastic forcing contributions are $\eta_{m}(\lambda, \phi ; t)$ and $\eta_{a}(\lambda, \phi ; t)$ in Eq. (3). Stochastic forcing is introduced to the temperature equation to simulate heat-sources contributed by, for example, convective type processes ranging from clouds to sloping convection or super cells, which include both heating and cooling terms due to release of latent heat and evaporation (additive) and due to the fluctuating horizontal mass flux as part of the heat flux convergence (multiplicative). The inclusion of a random forcing term to the model equations is quite common in modeling to incorporate subgrid-scale dynamics in the model or to ensure a more realistic model climate (Palmer, 2001). Note that for a given $(\lambda, \phi ; t)$, the same value of noise is used in all vertical $\sigma$ levels. The properties of the spatiotemporal correlated noise used in this paper are described in the Appendix.

\section{Experimental design and methods of analysis}

PUMA with zonally symmetric forcing is employed for all our simulations using the same vertical (five levels) and horizontal (T21) resolution, time stepping (one hour) and all other parameters. The simulations are run for 14 years with the last 3 years being subjected to analysis.

Experimental design: Two kinds of experiments are carried out: First, a simulation of 14 years is used as the unperturbed reference or control experiment. Then the ensemble experiments are performed with an ensemble consisting of $M=30$ members. Two type of ensembles were generated; (i) only perturbing the initial field, and (ii) both stochasti-

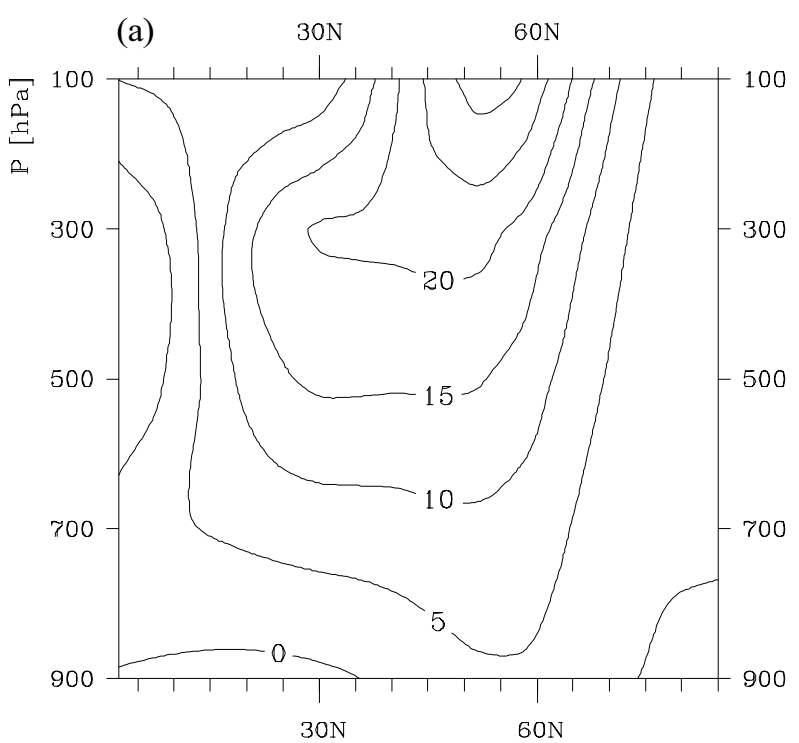

(b)

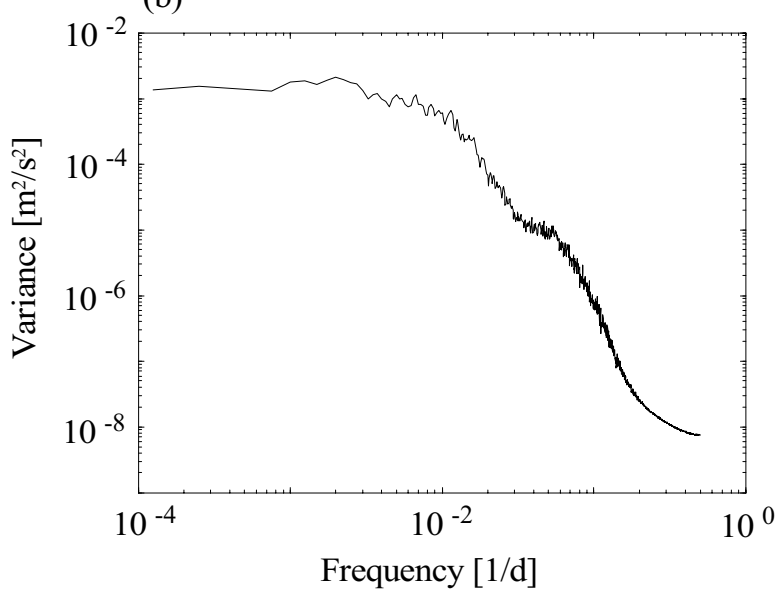

Fig. 1. Northern hemisphere cross section of the zonal and time mean zonal wind $(\mathrm{m} / \mathrm{s})(\mathbf{a})$, and power spectrum of the global mean relative vorticity (b)

cally perturbing the initial conditions and the model physics. In both experiments, the initial field was generated by adding a white noise of constant amplitude $I_{R}$ to the $\left(\ln p_{s}\right)$ initial field in Eqs. (1-5). This perturbation method does not produce dynamically constrained perturbed initial conditions as the breeding technique used at NCEP, or the singular vector approach used at ECMWF (Buizza et al., 1999; Molteni et al., 1996; Toth and Kalnay, 1993), rather it is designed to approximate a random sample from the initial probability distribution (Houtekamer and Derome, 1995). Still, this procedure will be shown below to describe adequately the ensemble. Stochastic physics was introduced in Eq. (3) as described in the previous Section and in this work, we study the effects of spatiotemporal correlated noise by keeping the noise dispersion $G(0,0)$ constant while varying its correlation time $\tau$ and correlation length $\ell$ (see Appendix for details). 

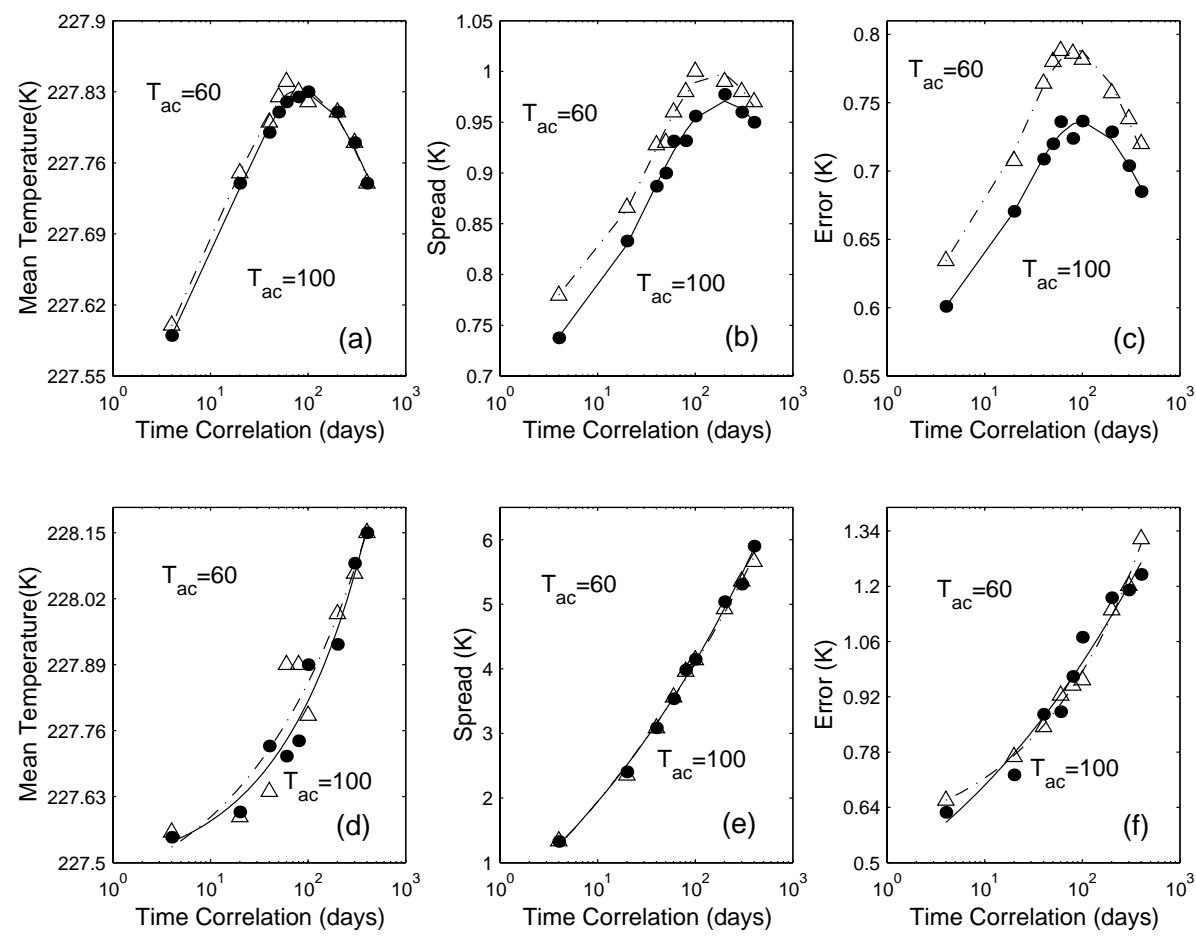

Fig. 2. Scores as a function of the correlation time $\log _{10} \tau$ for two different values of the annual cycle period $\left(T_{a c}=100(\right.$ circles $)$ and $T_{a c}=60$ (triangles)) for multiplicative (a-c) and additive (d-f) contributions of the noise. Lines show a fitting to a log-normal function for the multiplicative case, while for the additive noise the fitting corresponds to a power function. Noise parameters: $\ell=67.2$ deg, $\chi=10^{-1}(\mathrm{a}-\mathrm{c})$ and $\chi=10^{-4}(\mathrm{~d}-\mathrm{f})$.

Methods of analysis:

The most widely used measures for ensemble verification are the mean error and spread (Whitaker and Lougue, 1998; Wilks, 1995). The ensemble mean error is defined as the distance between the control run and the mean of the perturbed ensemble, that is,

Error $=\left\langle\left|\psi_{c}-\bar{\psi}\right|\right\rangle$

where $\bar{\psi}=(1 / M) \sum_{k=1}^{M} \psi_{k}$ is the ensemble average of $M$ members and $\psi_{c}$ is the deterministic/control trajectory calculated for each set of PUMA parameters without noise. In the following, the spatial average of this mean value has also been used which is defined as,

Mean $=\left\langle\frac{1}{M} \sum_{k=1}^{M} \psi_{k}\right\rangle$

The spread is defined to be the average rms distance between the ensemble members $k$ and the ensemble mean,

Spread $=\left\langle\left[\frac{1}{M} \sum_{k=1}^{M}\left(\psi_{k}-\bar{\psi}\right)^{2}\right]^{1 / 2}\right\rangle$

The angled brackets in the above definitions stand for the spatial average over all cells in the array and a time average over the last 3 years of the simulation. Results are independent of the period of time of average and similar results, although not shown here, were obtained for a single point
Table 1. Mean, spread and error scores for two values of the annual cycle $T_{a c}$ for ensembles based only on random perturbations to the initial field of PUMA

\begin{tabular}{cccc}
\hline$T_{a c}$ & Mean & Spread & Error \\
\hline 60 & 227.54 & 0.72 & 0.63 \\
100 & 227.53 & 0.70 & 0.59 \\
\hline
\end{tabular}

of the grid. For the PUMA model, the $300 \mathrm{hPa}$ temperature field was used to measure the scores of the ensemble. Higher values of the scores were obtained at higher pressure levels, not modifying the qualitative observed behavior. That is, the scores (ensemble mean, spread and error) refer to time (years 12 to 14) and space averages ( $300 \mathrm{hPa}$ temperatures on 2048 gridpoints).

\section{Results}

Ensembles generated by only perturbing the initial field of PUMA do not show relevant differences for the forecast scores values as $I_{R}$ is changed. Table 1 shows the forecast scores $^{2}$ obtained for two values of the annual cycle $T_{a c}$ for

\footnotetext{
${ }^{2}$ For this experiment, the control trajectory was considered to be one of the members of the ensemble.
} 


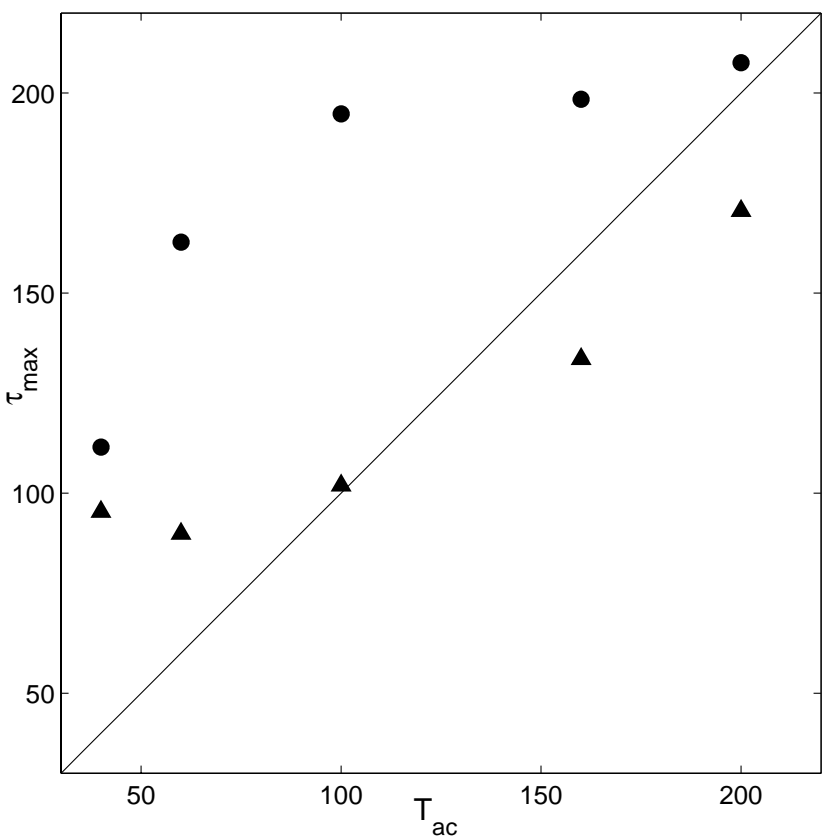

Fig. 3. Time correlation for which the maximum values of spread (circles) and error (triangles) are attained, $\tau_{\max }$, as a function of the annual cycle period $T_{a c}$.

$I_{R}$ within the range $\left[10^{-5}, 10^{-2}\right]$. Since PUMA equations correspond to a simplified physics of the atmosphere, mostly any random initial field gives rise to similar mean forecasted fields. The initial perturbations have small amplitudes compared to the natural variability and affect only the spinup phase of the model. Thus, from now on, we will concentrate on the behavior of PUMA when including stochastic physics while keeping constant $I_{R}=10^{-3}$.

The behavior of the ensemble mean, spread and error as a function of the correlation time is shown in Fig. 2 for two different values of the annual cycle period, $T_{a c}$, both when multiplicative $(\mathrm{a}-\mathrm{c})$ and additive $(\mathrm{d}-\mathrm{f})$ contributions of the noise are considered. The most remarkable effect is the occurrence of a local maximum value of the scores for the multiplicative noise case $\left(\eta_{m} \neq 0 ; \eta_{a}=0\right)$ for some intermediate values of the time correlation $\tau=\tau_{\max }$ and constant length correlation and noise dispersion. This peak seems to be the signature of a resonant behavior between the annual cycle period $T_{a c}$ and the noise correlation time as it will be shown later. For the limit case $\tau \rightarrow 0$ and finite $G(0,0)$, the noise intensity tends to zero and the noise vanishes (Lorenzo et al., 2003). In this way, the ensemble reduces to the case when only different random initial conditions were considered without stochastic physics, and the forecast scores tend to those calculated then (see Table I). Note that, strictly speaking, in the simulations it is not possible to attain this limiting case $\tau=0$. But the smaller the time step, the closer one gets to that limit. On the other hand, for $\tau \rightarrow \infty$ a kind of quenched or static Gaussian noise is obtained. In this case, relevant fluctuations of the noise are infrequent and their effects on the system are small (García-Ojalvo and Sancho, 1999).
For the additive case $\left(\eta_{a} \neq 0 ; \eta_{m}=0\right)$, Fig. $2 \mathrm{~d}-\mathrm{f}$, all the scores are observed to increase with $\tau$ and no resonance-like behavior was obtained. Moreover, the range of values of the spread is six times larger than for the multiplicative case (one and a half for the error), for both values of the annual cycle period $T_{a c}$, although here the noise variance is three orders of magnitude smaller than for the first case. Note that only for the limit $\tau \rightarrow 0$ the ensemble based only on random perturbations to the initial field is recovered. For the farthest limit, the scores are the highest obtained.

The resonant behavior observed for the multiplicative case has been analyzed in detail in Fig. 3. The values of the time correlation for which the spread and error attain a maximum value, $\tau_{\max }$, are shown as a function of the annual cycle pe$\operatorname{riod} T_{a c}$. Note that the maximum of the spread is displaced to higher values of the correlation time than for the error. Besides, for both scores, $\tau_{\max }>T_{a c}$, except for high $T_{a c}$ values where $\tau_{\max } \simeq T_{a c}$. Although the annual cycle period can be considered to be the most important time scale in the model, the presence of stochastic physics, as well as other time scales involved in the equations displace the time scale at which resonance takes place.

This behavior can be explained by using simple arguments derived from a toy model. If we admit that temperature dynamics is mainly conditioned by the annual cycle frequency $\left(\omega_{a c}=2 \pi / T_{a c}\right)$ and no spatial dependence is assumed, we can simplify enormously the thermodynamic Eq. (3) to the harmonic oscillator differential equation. Damping is considered to account for energy dissipation in the model. Noise forcing can be written in a more simplified way, in terms of a periodic forcing $\cos \left(\omega_{2} t\right)$, such that the "quenched" limit is obtained if $T_{2}=2 \pi / \omega_{2} \rightarrow \infty^{3}$. Then,

$\frac{d^{2} x}{d t^{2}}+2 \gamma \frac{d x}{d t}+\left[1+\alpha \cos \left(\omega_{2} t\right)\right] \omega_{a c}^{2} x=\beta \cos \left(\omega_{2} t\right)$

and the multiplicative case is obtained if $(\alpha \neq 0 ; \beta=0)$, while the additive case occurs if $(\alpha=0 ; \beta \neq 0)$. For this simplified model, amplitude resonance occurs for $\omega_{2}<\omega_{a c}$ independently of the considered forcing, although the resonant peak is much stronger for $\beta=0$, than for the additive case $(\alpha=0)$. As well, for $T_{2} \rightarrow \infty$, the mean value $\bar{x}>0$ for $\alpha=0$, while $\bar{x} \rightarrow 0$ for $\beta=0$. Thus, extrapolating these results to the original Eq. (3), the quenched additive noise gives rise to an overall increase of $T^{\prime}$, finally masking the resonance that was already smaller than for the multiplicative case. Although Eq. (12) represents a simplified model of the PUMA thermodynamic equation, it shows that resonance takes place at values close to the natural frequency of the oscillator as the damping and periodic forcings shift the resonant frequency. For the stochastic harmonic oscillatory system an average frequency $\overline{\omega_{2}}$ can be quantified for the additive case such that $\overline{\omega_{2}}<\omega_{a c}$ by some factor that depends on the damping force and the time correlation of the noise (Callenbach et al., 2002). Besides, it can be shown

\footnotetext{
${ }^{3}$ Moreover, as $T_{2} \rightarrow \infty$ both the cosine function and the time correlated noise show a "similar" time dependence.
} 

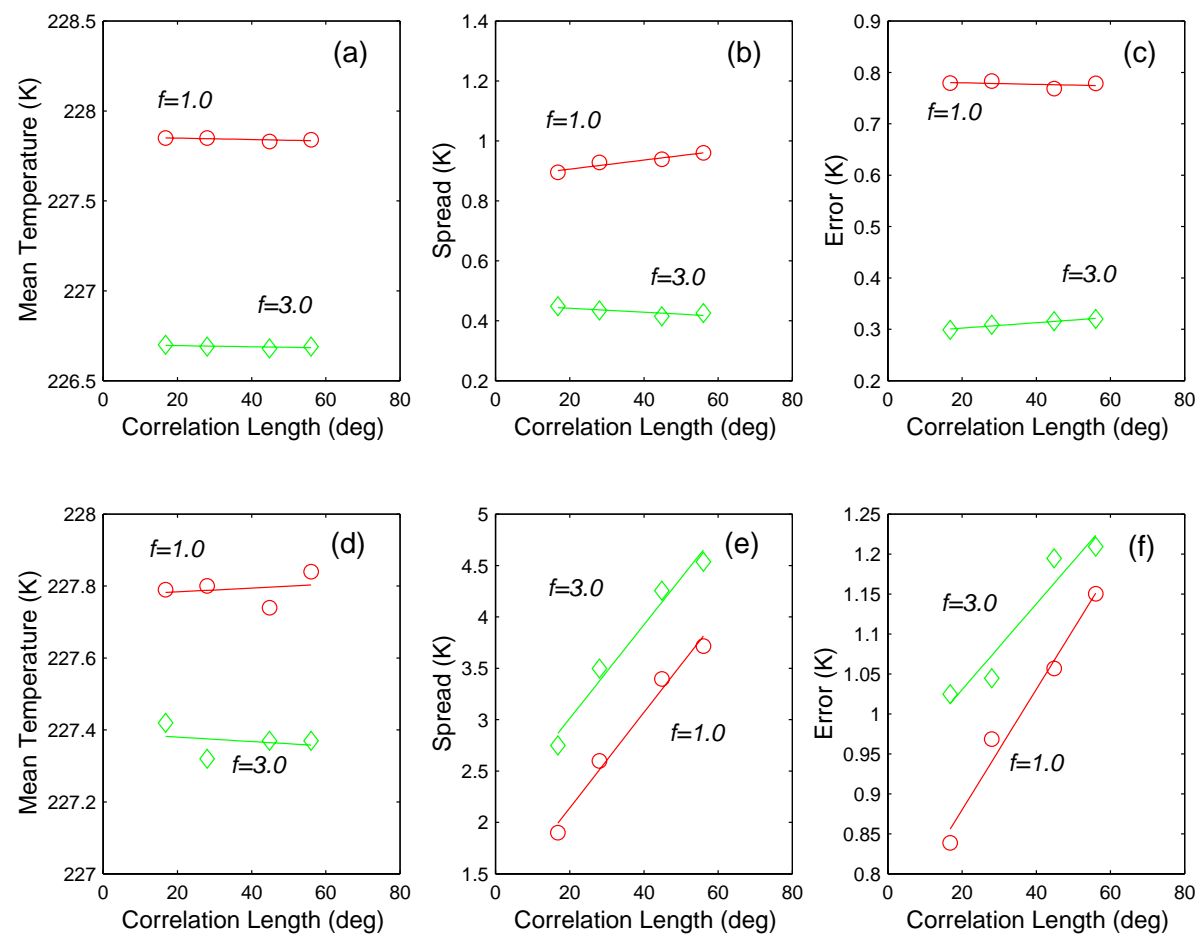

Fig. 4. Scores as a function of the the length correlation for two different values of the planetary vorticity $f$ for multiplicative (a-c) and additive (d-f) contributions of the noise. $T_{a c}=60$ days. Noise parameters: $\tau=100$ days, $\chi=10^{-1}(\mathrm{a}-\mathrm{c})$ and $\chi=10^{-4}(\mathrm{~d}-\mathrm{f})$.

that in this case, resonance does not take place (Masoliver and Porrá, 1993). As far as we now, the multiplicative case has not been solved analytically but numerical simulations qualitatively agree with those shown above for the periodic forcing.

The effect of the correlation length is analyzed in Fig. 4 for a constant value of the correlation time and noise intensity for two different values of the planetary vorticity $f$. Here again, the effect of multiplicative $(\mathrm{a}-\mathrm{c})$ or additive $(\mathrm{d}-\mathrm{f})$ contributions of the noise is analyzed. Although few resonant cases with changing spatial correlation scale $\ell$ have been described in the literature (Lorenzo et al., 2003; Santos and Sancho, 2001), we expected here to obtain different natural length scales as the vorticity changes and analyze its effect on the scores. Unfortunately, only a linear increase of the scores with the correlation length is obtained, although this is still a nontrivial effect, as for the trivial case (i.e. noise dispersion is not kept constant as $\ell$ is varied, Eq. A4) a decreasing of the scores with $\ell$ should be expected (Lorenzo et al., 2003). Spread and error increase faster with $\ell$ for the additive than for the multiplicative case. For a constant value of $\ell$, spread and error decrease with increasing $f$, Fig. 4a-c, while the opposite behavior is observed for the additive case $(\mathrm{d}-\mathrm{f})$. Note that for any value of $\ell$ the mean temperature increases with decreasing planetary vorticity. The increase in the mean temperature at $300 \mathrm{hPa}$ level is explained by the conservation of potential vorticity. If the planetary vorticity decreases, the height of the column between the same isentropic surfaces rises and the mean temperature at this level increases.
The values of the ensemble scores shown in Fig. 2 and Fig. 4 depend on the noise dispersion. In order to simplify the discussion, we will focus only in the behavior of the spread for the additive case, since the remaining scores behave similarly. Figure 5 shows the spread for some constant value of $\ell$ and $\tau$ as a function of the noise dispersion. The spread increases with noise dispersion as can be expected. It is worth remarking that for the multiplicative case, the resonant peak shown in Fig. 2 becomes wider around $\tau_{\max }$, as predicted in (Lorenzo et al., 2003).

Finally, the sensitivity of an ensemble on the number $M$ of its members is analyzed in Fig. 6. In all cases, a rapid convergence to a nearly constant value (independent of $M$ ) is attained. Moreover, the ensemble members span the range of uncertainty of the error independently of the ensemble size $M$.

\section{Conclusions: implications for ensemble weather fore- casting}

We have analyzed separately the systematic nontrivial effects of the different noise parameters, intensity $\chi$, correlation time $\tau$ and length $\ell$, in the PUMA. Spatiotemporal correlated noise is introduced into the thermodynamic energy equation with multiplicative or additive contributions. In both cases, the fact of including stochastic physics in the model gives rise to higher forecast scores values than using only an ensemble based on random perturbations to the initial conditions. For the multiplicative spatiotemporal correlated noise, a resonant 


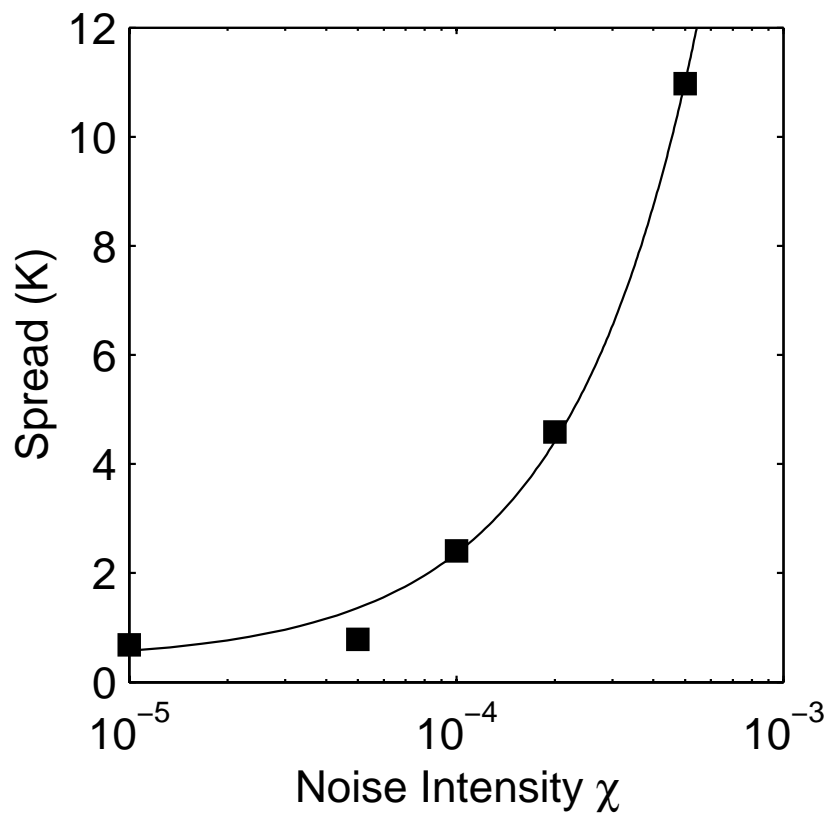

Fig. 5. Ensemble spread as a function of the noise intensity $\chi$ when the additive contribution of noise is considered. $T_{a c}=60$ days. Noise parameters: $\tau=20$ days, $\ell=67.2 \mathrm{deg}$ and $\chi=10^{-4}$.

behavior between the natural time scale of the PUMA model and the noise time correlation $\tau$ seems to be the responsible for the observed maxima of the score measures (ensemble mean, spread and 'error'). Correlated stochastic forcing lead us to obtain the natural time scales for which the largest spread could be obtained without increasing the noise intensity.

The observed resonant effect is very sensitive to the place where noise is inserted into the equations, as well as to the natural time or length scales of the model. Regarding the first, much work is still to be done in modeling the best place into the equations where the most effective values of the scores could be obtained. Only exhaustive numerical simulations seem to work. On the other hand, the presence of large length scales into the system makes difficult to obtain some resonances since very large computer simulations are then needed in order to get some accuracy. Probably, this is the reason why we were unable to find any response to our spatially correlated noise forcing, since the most probably length scale of PUMA is well above the correlation lengths used in this paper. Nevertheless, previous experiments with more simple models lead us to expect that such resonance should occur (Lorenzo et al., 2003).

In our simulations, time and length scales of the noise forcing are chosen to maximize spread when a resonance with the natural time and length scales of the model occur. This approach is different to the 'stochastic optimals' method (Kleeman and Moore, 1997; Moore and Kleeman, 1999), which represents the spatial patterns onto which stochastic forcing must project to maximize error growth over a given time interval. This method bears resemblance to the recently intro-

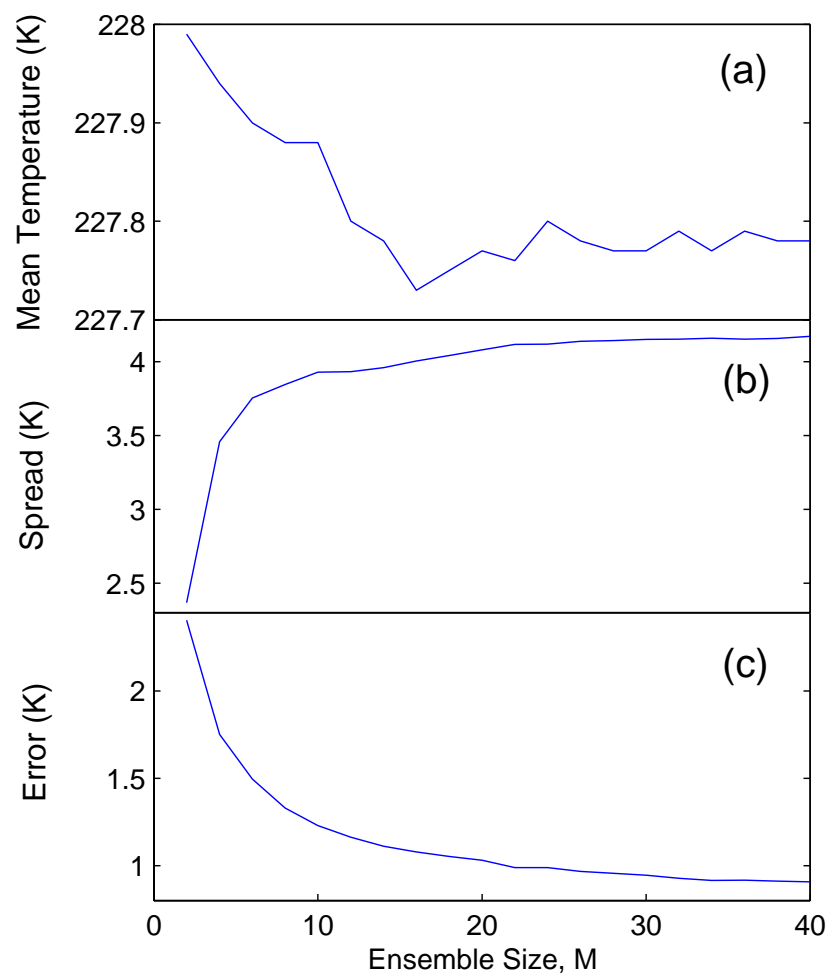

Fig. 6. Ensemble mean (a), spread (b) and error (c) as a function of the ensemble size $M . T_{a c}=60$ days. Noise intensity for the additive case $\chi=10^{-4}$.

duced 'forcing singular vectors' technique (Barkmeijer et al., 2003), but does not allow for time-dependent noise forcings. In fact, our work is closer to the new scheme of ensemble prediction introduced in 1998 at the ECMWF to simulate random model errors due to parameterized physical processes (Buizza et al., 1999). This scheme is based on the notion that this randomness is coherent between the different parameterization modules and has a certain coherence on the space and time scales represented by the model (Houtekamer et al., 1996; Mylne et al, 2002). Looking for this coherence in terms of resonances was the purpose of the work presented here.

Finally, the results derived here were made using a relatively simple global circulation model, far from the more sophisticated meteorological models used for weather and seasonal forecasts where other scales and parameterizations are included. The results shown here concern mean values over several years, which are closer to seasonal forecast than to weather forecast which it requires only ensemble averages at a leading time. Our method still requires to optimize the stochastic physics in terms of which equations need to be forced to maximize the spread and the role played by the different scales involved in a more complex meteorological model and how they affect to the predictability of the model. In this case, our methods shown here should be accompanied by an additional theoretical framework as it was shown for example by Moore and Kleeman (1999). Despite these reser- 

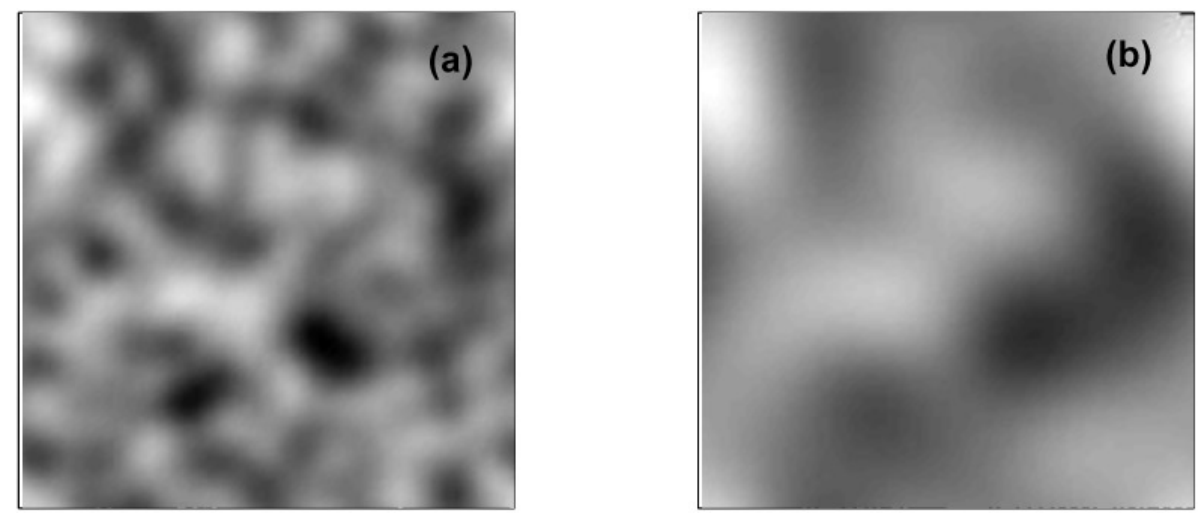

Fig. 7. Patterns of noise for two different values of the correlation length. (a) $\ell=39.2 \mathrm{deg}$ and (b) $\ell=112$ deg for a grid consisting of $32 \times 64$ points. $\tau=20$ days and $\chi=10^{-4}$

vations, we are confident that the results discussed here could be used for ensemble weather/seasonal forecasts. However, when transferring our results one has to realize that most ensemble predictions show deficiency forecast variance. But the goal in ensemble applications is not simply to increase the spread but rather increase the right kind of variance. That is, ensemble variability must represent the actual forecast error. This includes the proper covariance comparing ensemble forecasts and the verification, which requires the use of ensemble and probabilistic verification methods, which are not used here.

\section{Appendix Stochastic forcing}

The stochastic forcing used in the PUMA model corresponds to a Gaussian spatiotemporal distributed noise with zero mean whose correlation function is

$$
\begin{aligned}
\left\langle\eta(x, t) \eta\left(x^{\prime}, t^{\prime}\right)\right\rangle & =G\left(\left|x-x^{\prime}\right|,\left|t-t^{\prime}\right|\right) \\
& =C\left(\left|x-x^{\prime}\right| / \ell\right) \gamma\left(\left|t-t^{\prime}\right| / \tau\right),
\end{aligned}
$$

The temporal correlation function, $\gamma(s / \tau)$, describes an Ornstein-Uhlenbeck (OU) process given by,

$$
\gamma(s / \tau)=\frac{1}{\tau} \exp (-s / \tau)
$$

where $\tau$ corresponds to the correlation time for the OU process. The spatial correlation is,

$C(s / \ell)=\frac{\chi^{2}}{2 \pi \ell^{2}} \exp \left(-s^{2} / 2 \ell^{2}\right)$

where $\chi^{2}$ is the noise intensity and $\ell$ is the correlation length.

In order to study the nontrivial effects of noise, the noise dispersion,

$G(0,0)=\frac{\chi^{2}}{2 \pi \tau \ell^{2}}$

is kept constant, while varying $\tau$ or $\ell$. Details on the numerical generation of a spatiotemporal correlated noise given by Eqs. (A2)-(A3) are given in (Alonso et al., 2002) and (García-Ojalvo and Sancho, 1999). We would like to emphasize that for this numerical method, neither correlation length values smaller than 3 grid points, nor larger than half of the grid, can be used in order to guarantee some accuracy for the results.

The independent role of the three noise parameters (intensity, time and length correlations) has been analyzed in this paper. Figure 7 shows two patterns of noise for two different values of $\ell$ that are used into the simulations. Note the different size of patches as $\ell$ increases.

Acknowledgements. We want to thank S. Alonso and J. M. Sancho for providing us with some of the noise routines used in our numerical simulations, as well as for a careful reading of the manuscript. This work was supported by Xunta de Galicia (Spain) under Research Grant No. PGIDT01MAM20802PR.

\section{References}

Alonso, S., Sagués, F. and Sancho, J. M.: Excitability transitions and wave dynamics under spatiotemporal structured noise, Phys. Rev. E, 65, 066107(1)-066107(11), 2002.

Anderson, J., van den Dool, H., Barnston, A., Chen, W., Stern, W. and Ploshay, J.: Present-day capabilities of numerical and statistical models for atmospheric extratropical seasonal simulation and prediction, Bull. Am. Meteorol. Soc., 80, 1349-1361, 1999.

Barkmeijer, J., Iversen, T., and Palmer, T. N.: Forcing singular vectors and other sensitive model structures, Q.J.R. Meteorol. Soc., 129, 2401-2423, 2003.

Buizza, R., Miller, M., and Palmer, T. N.: Stochastic representation of model uncertainties in the ECMWF Ensemble Prediction System, Q.J.R. Meteorol. Soc., 125, 2887-2908, 1999.

Callenbach, L., Hänggi, P., Linz, S. J., Freund, J. A., and Schimansky-Geier, L.: Oscillatory systems driven by noise: Frequency and phase synchronization, Phys. Rev. E, 65, 051110(1)051110(11), 2002.

Cane, M. A., Zebiak, S. E., and Dolan, S. C.: Experimental forecasts of El Niño, Nature, 321, 827-832, 1986.

DelSole, T. M. and Farrell, B. F.: A stochastically excited linear system as model for quasigeostrophic turbulence: analytic results 
for one- and two-layer fluids, J. Atmos. Sci., 52, 2531-2547, 1995.

Farrell, B. F. and Ioannou, P. J.: Stochastic dynamics of baroclinic waves, J. Atmos. Sci., 50, 4044-4057, 1993.

Fraedrich, K. and Ziehmann-Schlumbohm, C.: Predictability experiments with persistence forecasts in a red-noise atmosphere, Q.J.R. Meteorol. Soc., 120, 387-428, 1994.

Fraedrich, K., Kirk, E., and Lunkeit, F.: Portable University Model of the Atmosphere, Deutsches Klimarechenzentrum, Report No. 16, 1998. "http://puma.dkrz.de/puma".

García-Ojalvo, J. and Sancho, J. M.: Noise in spatially extended systems, Springer-Verlag, New-York, 1999.

Harrison, M. S. J., Palmer, T. N., Richardson, D. S., and Buizza, R.: Analysis and model dependencies in medium-range ensembles: Two trasplant case studies, Q.J.R. Meteorol. Soc., 125, 24872516, 1999.

Hasselmann, K.: Stochastic climate models. Part I. Theory, Tellus, 28, 473-485, 1976.

Hoskins, B. J. and Simmons, A. J.: A multi-layer spectral method and the semi-implicit method, Q.J.R. Meteorol. Soc., 101, 637$655,1975$.

Houtekamer, P. L. and Derome, J.: Methods for ensemble prediction, Mon. Wea. Rev., 123, 2181-2196, 1995.

Houtekamer, P. L., Lefaivre, L., Derome, J., Ritchie, H., and Mitchell, H.: A system simulation approach to ensemble prediction, Mon. Wea. Rev., 124, 1225-1242, 1996.

Kalnay, E.: Atmospheric modeling, data assimilation and predictability, Cambridge Univ. Press, UK, 2003.

Kleeman, R. and Moore, A. M.: A theory for the limitation of ENSO predictability due to stochastic atmospheric transients, J. Atmos. Sci., 54, 753-767, 1997.

Lorenzo, M. N. and Pérez-Muñuzuri, V.: Colored noise-induced chaotic array synchronization, Phys. Rev. E, 60, 2779-2787, 1999.

Lorenzo, M. N. and Pérez-Muñuzuri, V., Influence of low intensity noise on assemblies of diffusively coupled chaotic cells, Chaos, 11, 371-376, 2001.

Lorenzo, M. N., Santos, M. A., and Pérez-Muñuzuri, V.: Spatiotemporal stochastic forcing effects in an ensemble consisting of arrays of diffusively coupled Lorenz cells, Chaos, 13, 913-920, 2003.
Lunkeit, F.: Synchronization experiments with an atmospheric global circulation model, Chaos, 11, 47-51, 2001.

Masoliver, J. and Porrá, J. M.: Harmonic oscillators driven by coloured noise: Crossovers, resonances, and spectra, Phys. Rev. E, 48, 4309-4319, 1993.

Molteni, F., Buizza, R., Palmer, T. N., and Petroliagis, T.: The ECMWF ensemble prediction system: Methodology and validation, Q.J.R. Meteorol. Soc., 122, 73-120, 1996.

Moore, A. M. and Kleeman, R.: Stochastic forcing of ENSO by intreseasonal oscillation, J. Climate, 12, 1199-1220, 1999.

Mylne, K. R., Evans, R. E., and Clark, R. T.: Multi-model multianalysis ensembles in quasi-operational medium-range forecasting, Q.J.R. Meteorol. Soc., 128, 361-384, 2002.

Newman, M., Sardeshmukh, P. D., and Penland, C.: Stochastic forcing of the wintertime extratropical flow, J. Atmos. Sci., 54, 43545, 1997

Palmer, T. N.: A nonlinear dynamical perspective on model error: A proposal for non-local stochastic-dynamic parametrization in weather and climate prediction models, Q.J.R. Meteorol. Soc., 127, 279-304, 2001.

Penland, C.: A stochastic model of IndoPacific sea surface temperature anomalies, Physica D, 98, 534-558, 1996.

Pitcher, E.: Application of stochastic dynamic prediction to real data, J. Atmos. Sci., 34, 3-21, 1977.

Santos, M. A. and Sancho, J. M.: Front dynamics in the presence of spatiotemporal noises, Phys. Rev. E, 64, 016129(1)-016129(11), 2001.

Smith, L. A., Ziehmann, C., and Fraedrich, K.: Uncertainty dynamics and predictability in chaotic systems, Q.J.R. Meteorol. Soc., 125, 2855-2886, 1999.

Toth, Z. and Kalnay, E.: Ensemble forecasting at NMC: The generation of perturbations, Bull. Amer. Meteor. Soc., 74, 2317-2330, 1993.

Whitaker, J. S. and Lougue, A. F.: The relationship between ensemble spread and ensemble mean skill, Mon. Wea. Rev., 126 , 3292-3302, 1998

Wilks, D. S.: Statistical methods in the atmospheric sciences, Academic Press, New-York, 1995.

Ziehmann, C.: Comparison of a single-model EPS with a multimodel ensemble consisting of a few operational modes, Tellus, 52A, 280-299, 2000 